\title{
Clinical findings in determining referral criteria for coronavirus disease (COVID-19) patients at a makeshift isolation center of Bekasi, West Java
}

\author{
Stefi Geovani Valentin Hayon, ${ }^{*}{ }^{*}$ Florentina Priscilia, ${ }^{1}$ and Hariri ${ }^{2}$ \\ ${ }^{1}$ General Practitioner, Makeshift COVID-19 Isolation Center of Bekasi District, Bekasi, Indonesia \\ ${ }^{2}$ Epidemiologist, Bekasi District Health Office, Bekasi, Indonesia
}

\section{KEYWORDS} COVID-19

Isolation center Referral criteria Early warning signs

\begin{abstract}
Coronavirus disease (COVID-19) has become a pandemic concern with the clinical manifestations range from asymptomatic to severe organ failure. Early contact tracing and isolation are important keys to slow the infection spread within communities. Clinical triage and early recognition of warning signs on admission are needed to classify patients based on clinical severity. Due to the limited capacity of hospital settings for COVID-19 patients, a makeshift isolation center is needed for all confirmed and suspected cases with mild or no symptoms who will be provided with basic medical care, frequent monitoring, and rapid referrals. During observations, the physician needs to raise early alertness of the patient who is at risk to develop a poor outcome. This study reports five cases of patients who came with mild or no symptoms at initial presentation and developed into worsening disease progression after several days of observation. These patients developed shortness of breath, fatigue, abdominal disturbances, and chest pain. These findings were supported by the physical examinations that showed a decrease in blood oxygen saturation. Similarly, with the Centers for Disease Control and Prevention (CDC) criteria for the early warning signs, these patients were referred to the hospital-based isolation setting that could provide more advanced and optimal management. This study describes clinical manifestations of COVID-19 patients to decide the ideal time for referral.
\end{abstract}

(c) The Journal 2021. This article is distributed under a Creative Commons Attribution-ShareAlike 4.0 International license.

\section{Introduction}

Severe acute coronavirus 2 (SARS-CoV-2) is a novel pathogen identified as an enveloped ribonucleic acid (RNA) betacoronavirus. This pathogen causes coronavirus disease (COVID-19) and was confirmed to spread through respiratory droplets when talks, coughs, or sneezes. The clinical manifestations range from asymptomatic to severe organ failure, infecting especially the lungs, brain, intestines, and heart. ${ }^{1}$ The organ failure is caused by a hyperinflammatory process due to massive cytokine release and lymphohistiocytosis, called a "cytokine storm". The host immune response to the SARS-CoV-2 results in an excessive inflammatory reaction, since there is an increase in cytokine and various immune cells which damage vascular, capillary, alveolar, and organs. This

*Correspondence: stefi.geovani.v@mail.ugm.ac.id Makeshift COVID-19 isolation center, Bekasi District, Indonesia condition of COVID-19 progression will also give a symptom of hemodynamic instability that results in the development of hypoxia. ${ }^{2,3}$

COVID-19 has spread globally and become a pandemic. Until February 2021, SARS-CoV-2 has infected 113 million people around the world causing more than 2 million deaths. ${ }^{4}$ In Indonesia, the cases were still increasing in September 2020. The total cases reached around 200,000 people at the highest peak. ${ }^{5}$ As the cases continued to rise, most of the hospitals were overwhelmed due to the limited number of available beds. Responding to this condition, the local government, especially where the cases were locally transmitted, released an official statement to create a makeshift isolation center. Whether it is hospital-based or in nonhospital based health facilities, this isolation center is a key factor for successful transmission control. A comparative study by Dickens et al. (2020) found that the institution-based isolation made contact rates 
reduced approximately $75 \%$ in the household and by $90 \%$ in the community, whereas contact rates in home-based isolation were reduced by $50 \%$. A similar finding in a study by Chen et al. (2020) described how the isolation centers in Wuhan were established for all infected and potentially exposed individuals. These isolation centers provided triage, basic medical care, frequent monitoring, rapid referrals, in addition to essential living and social engagements for the wellbeing of those isolated. From this system, the risk of within-household transmission was reduced significantly. ${ }^{6,7}$ The crucial part of these makeshift isolation centers is the effective and efficient triage to classify the patients based on their recent condition. Due to the emergency setting, the lack of adequate resources to evaluate a patient's disease progression objectively might be a challenge during isolation since there is commonly no laboratory or radiology examination for this setting. The physician needs to make a clear judgement based on daily clinical findings and know when to refer a patient before any deterioration happens. Based on the World Health Organization (WHO) algorithm for COVID-19 triage and referral, mild cases are referred to community care and an isolation center. If the condition becomes deteriorated, the patients should be referred to the higher level of facilities. The WHO recommends criteria for referral based on physician judgements and local capacity. The criteria is respiratory failure, altered mental status, shortness of breath, respiratory rate $>30$ times/minute, systolic blood pressure $<90 \mathrm{mmHg}$, organ failure and oxygen saturation $\mathrm{SpO} 2<94 \% .{ }^{8}$ This study aims to describe the clinical manifestations of COVID-19 patients who were observed and needed to be referred from makeshift isolation settings. Moreover, it provides simple referral criteria based on clinical manifestations in a makeshift isolation center for COVID-19 patients.

\section{Case illustration}

The first patient is a 39-year-old male who was referred from a local primary health care in Bekasi, Indonesia to a makeshift COVID-19 isolation center. He came with the main symptom of productive cough for 3 days before admission accompanied by a sore throat, myalgia, and headache, but the physical examination found no abnormality. The patient claimed that he had no contact with suspected or confirmed COVID-19 patients; otherwise, he worked at a public service office where he was required to have contact with many people and worked the night shifts 5 days a week. The patient had a history of hyperuricemia and was undergoing treatment with allopurinol. One day after the admission $\left(4^{\text {th }}\right.$ day of symptom onset), he complained of worse cough, excessive sweating, fatigue, and anorexia. By the physical examination, the blood pressure was $150 / 90 \mathrm{mmHg}$, respiratory rate 20 times/minute, heart rate 92 times/minute, and oxygen saturation $98 \%$ in room air. The next day ( $5^{\text {th }}$ day of symptom onset), he felt the same symptoms with the addition of chest pain, shortness of breath, nausea, and abdominal discomfort. The physical examination showed a sub-febrile body temperature of $37.2^{\circ} \mathrm{C}$, blood pressure $110 / 80 \mathrm{mmHg}$, respiratory rate 24 times/minute, heart rate 90 times/minute, and decreased oxygen saturation within the range 93\%$95 \%$ on room air, with increased effort of breathing and bilateral rhonchi at the basal field of the lungs. He was confirmed with positive SARS-CoV-2 by reverse-transcription polymerase chain reaction (RT$\mathrm{PCR}$ ) test one day before the symptoms occurred. The patient was referred to the hospital after 2 days of observation ( $6^{\text {th }}$ day of the confirmed case).

The second patient is a 40-year-old male who was referred from a company health clinic in Bekasi, Indonesia with no symptoms and comorbidities. He was confirmed positive with SARS-CoV-2 by RT-PCR test one day before admission. The company doctor suggested the patient to be isolated at the makeshift isolation center for COVID-19 of Bekasi. On the day of admission and $1^{\text {st }}$ day of observation, he had no symptoms and physical examination results were in the normal range. On the $2^{\text {nd }}$ day of observation ( $3^{\text {rd }}$ day of the confirmed case), he felt chill, hard to fall asleep, myalgia, and dry cough but the physical examination found no abnormality. The next day ( $3^{\text {rd }}$ day of observation $/ 4^{\text {th }}$ day of the confirmed case), he complained about persistent symptoms with loss of appetite, worsening cough, and fever. By the physical examination, the physician found febrile body temperature within a range of $37.5^{\circ} \mathrm{C}$ and $37.8^{\circ} \mathrm{C}$, blood pressure was $120 / 80 \mathrm{mmHg}$, respiratory 


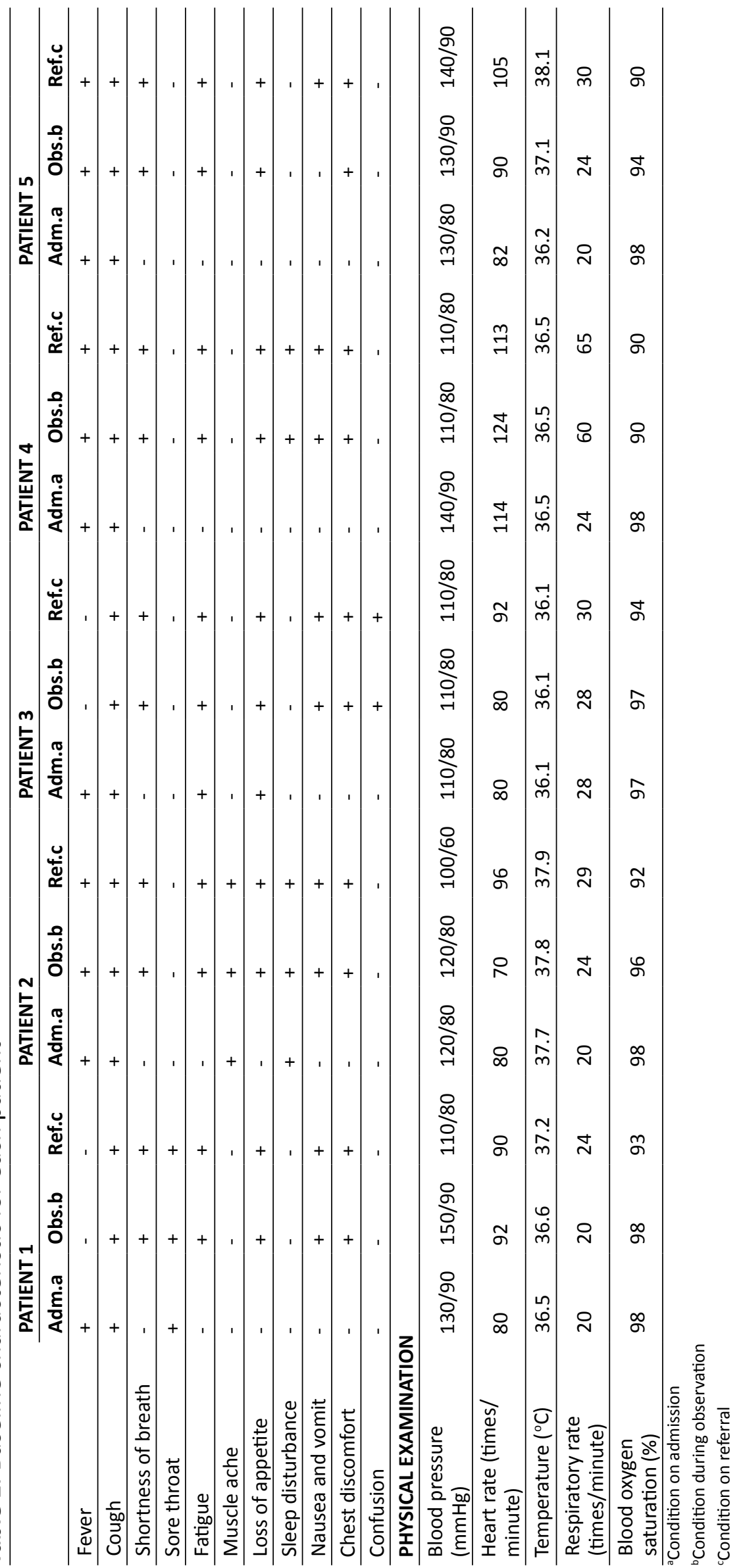


rate 24 times/minute, heart rate 70 times/minute, oxygen saturation $96 \%$ on room air, and normal lung sounds. The symptoms worsened with the difficulty of oral feeding and recurrent vomiting. On the $6^{\text {th }}$ day of observation ( $7^{\text {th }}$ day of the confirmed case), he felt extreme fatigue, chest pain, shortness of breath, and nausea. The physical examination showed that the body temperature was $37.9^{\circ} \mathrm{C}$, blood pressure $100 / 60 \mathrm{mmHg}$, respiratory rate 29 times/minute, heart rate 96 times/minute, and decreased oxygen saturation $92 \%$ in room air. The patient was referred to the hospital after 6 days of observation ( $7^{\text {th }}$ day of the confirmed case).

The third patient is a 62-year-old female who was referred from the local primary health care in Bekasi, Indonesia to the COVID-19 makeshift isolation center of Bekasi with symptoms of general weakness, dry cough, anorexia, and epigastric pain on the $6^{\text {th }}$ day of the confirmed case. These symptoms occurred 6 days before admission and got worse over time. She was confirmed SARS-CoV-2 by RT-PCR on the same day when the symptoms occurred. The patient had a history of pulmonary tuberculosis and hypercholesterolemia. On the day of admission, the physician found general weakness with a normal body temperature $\left(36.1^{\circ} \mathrm{C}\right)$, normal blood pressure $(110 / 80) \mathrm{mmHg}$, respiratory rate 28 times/minute, heart rate 80 times/minute, and oxygen saturation $97 \%$ in room air. Five hours after admission, she complained of chest pain, shortness of breath, nausea, and vomiting. The physician directly examined and found altered mental status, increased respiratory rate 30 times per minute, persistent oxygen saturation $95 \%$ on room air, and full rhonchi on the bilateral lung. The patient was immediately referred to the hospital on the day of admission ( $6^{\text {th }}$ day of the confirmed case).

The fourth patient is a 44-year-old male who was referred from the local primary health care in Bekasi, Indonesia to the COVID-19 makeshift isolation center of Bekasi with symptoms of dry cough and history of fever 3 days before confirmed. These symptoms had occurred for 7 days. The patient had no history of illness. On the day of admission, the physician found continuous cough and normal body temperature $\left(36.5^{\circ} \mathrm{C}\right)$, elevated blood pressure $(140 / 90) \mathrm{mmHg}$, respiratory rate 24 times/minute, heart rate 114 times/minute, and oxygen saturation $98 \%$ on room air. The next day, he complained about shortness of breath, loss of appetite, worsening dry cough, and hard to fall asleep. The physician directly examined and found increased respiratory rate of 60 times per minute and persistent oxygen saturation $90 \%$ in room air. The patient was immediately referred to the hospital on the next day of admission ( $5^{\text {th }}$ day of the confirmed case).

The fifth patient is a 56-year-old male who came with the main symptom of productive cough for 6 days before admission accompanied by fever. The patient had a history of hypercholesterolemia. One day after the admission, he complained of shortness of breath, fatigue, loss of appetite, nausea, and vomiting. In the physical examination, the blood pressure was $130 / 90 \mathrm{mmHg}$, body temperature of $37.1^{\circ} \mathrm{C}$, respiratory rate 24 times/minute, heart rate 92 times/minute, and oxygen saturation $94 \%$ in room air. He was confirmed positive with SARS-CoV-2 by RT-PCR test one day after the symptoms occurred. The patient was referred to the hospital after 1 day of observation ( $5^{\text {th }}$ day of the confirmed case) with decreased oxygen saturation to $90 \%$ and increased respiratory rate to 30 times/minutes.

The patients' baseline characteristics are described in Table 1, indicating the main symptoms include fever, cough, shortness of breath, sore throat, fatigue, muscle ache, loss of appetite, sleep disturbance, nausea and vomit, chest discomfort, and confusion. In addition, the patients' basic physical examinations are summarized for blood pressure, heart rate, temperature, and blood oxygen saturation which were examined regularly at the makeshift isolation center that has limited medical resources with no laboratory and radiology facilities. Based on those symptoms and clinical signs, the attending physicians could immediately figure out the warning signs for referral.

\section{Discussion}

This is a case series of five COVID-19 patients from a makeshift isolation center of Bekasi, Indonesia that were selected consecutively. Written informed consent was obtained from the patients for publication of study and they understand that their names and initials will not be published. The median 
age of the patients was 40 years old similar to a descriptive study conducted in Indonesia by Karyono et al. (2020) $(n=26,454)$ that showed the highest proportion of COVID-19 cases was in the age group of $31-45$ years $(29.3 \%)$. Another case series in the Indonesian population reported by Tenda et al. (2020) also had median age of 42 years old. Male patients were predominant in this study in accordance with Karyono et al. (2020) that described how COVID-19 infected more males $(54.6 \%, n=15,123)$ than females. ${ }^{9,10}$ A systematic literature review showed that the proportion of males was significantly higher in the critical or deceased group compared to the noncritical group. ${ }^{11}$

All patients came with a history of fever and developed a cough on admission that are primarily exhibited in COVID-19 patients with mild cases suitable to be treated at a makeshift isolation center based on the WHO algorithm for COVID-19 triage and referral. ${ }^{1,4,12}$ During the observations, other symptoms that mostly developed were loss of appetite, nausea and vomiting, chest discomfort, and shortness of breath with a decrease of blood oxygen saturation. Based on these findings, we suggest them as the initial warning signs to consider a referral decision as recommended by the Centers for Disease Control and Prevention (CDC). ${ }^{13}$ The warning signs are trouble of breathing, pain, or pressure in the chest, mental confusion, inability to stay awake, bluish lips, or white face. In our cases, all of the patients developed shortness of breath and chest pain while one patient also developed confusion. The decrease of blood oxygen saturation was consistent with the recent WHO algorithm of referral criteria. Based on those findings we suggest increasing the awareness of poor prognosis that might happen during observation in order to best know when to make a referral. A meta-analysis study found that patients who came with shortness of breath/dyspnea were more likely to develop into critical condition and had worse outcomes such as death. ${ }^{11}$ In our study, most of the patients did not complain about shortness of breath at the initial presentation, but we found that the blood oxygen saturation decreased. This condition might happen due to hypoxemia caused by SARS-CoV-2 that possibly has an idiosyncratic effect on the chemoreceptors of the carotid body.
Normally, hypoxemia condition sends a signal to the medulla oblongata to increase the respiratory center's output by activating the phrenic nerve and diaphragm. However, in COVID-19 patients, this signal is disrupted and fails to respond. ${ }^{13}$ Shortness of breath suggests poor lung function and lacking oxygen. Therefore, when the patient is found to have difficulty in breathing or no fever, it is necessary to be alert for further deterioration of the patient's condition.

The disease progression for each patient was notably similar that showed predominantly worsening on the $6^{\text {th }}$ day after the onset of symptoms. Based on an observational study by Chen et al. (2020), the disease progression during this time could be explained by uncontrolled viral replication ${ }^{7}$ and increased inflammatory response including CRP, ferritin, and pro-inflammatory cytokines which indicate the presence of a cytokine storm. ${ }^{2}$ The clinical features of this cytokine storm are the development of damaged tissues and organs since there are high immune responses, including fever, progression to pneumonia with symptoms of decreased oxygen saturation, liver damage, acute kidney, anemia, and impaired hematopoietic function. These conditions need special attention to be prepared for a referral to hospital-based isolation setting for the advanced medical treatment. ${ }^{15}$

We acknowledge that our study had some limitations. First, the study population was small and might not represent the vast clinical manifestations of COVID-19. Second, this study had limited data about the ancillary tests because the center does not have a comprehensive laboratory and radiology department for further assessment about patients' overall condition and prognosis.

\section{Conclusion}

From this case series, we describe the conditions of COVID-19 patients and suggest a comprehensive clinical observation for those who are admitted to a makeshift isolation center. Early warning signs based on clinical manifestations such as shortness of breath, fatigue, abdominal disturbances, and chest discomfort followed by decrease of blood oxygen saturation during isolation should be alerted 
for referral criteria in COVID-19 patients with mild cases. Despite having limited medical resources, we can consider clinical signs and symptoms to assess the disease progression within 7-10 days related to the pathophysiology of a cytokine storm for the same duration. These findings can help the attending physicians to consider a referral to better health facilities.

\section{Conflict of interests}

This study did not receive specific grants from funding agencies in the public sector, commercial, or non-profit section.

\section{References}

1. Huang R, Zhuid L, Xue L, Liu L, Yan X, Wang J, et al. Clinical findings of patients with coronavirus disease 2019 in Jiangsu Province, China: a retrospective, multi-center study. PLoS Negl Trop Dis [Internet]. 2020;14(5):1-14. Available from: $\quad h t t p: / / d x . d o i . o r g / 10.1371 /$ journal. pntd.0008280

2. Gao YM, Xu G, Wang B, Liu BC. Cytokine storm syndrome in coronavirus disease 2019: a narrative review. J Intern Med. 2020;(June):115.

3. Ye Q, Wang B, Mao J. The pathogenesis and treatment of the "Cytokine Storm" in COVID-19. J Infect. 2020;80:607-13.

4. World Health Organization. WHO coronavirus disease (COVID-19) dashboard [Internet]. 2021 [cited 2020 Sep 24]. Available from: https:// covid19.who.int/

5. Kemenkes RI. Infeki Emerging : Media Informasi Resmi Terkini Penyakit Infeksi Emerging [Internet]. 2020 [cited 2020 Sep 24]. Available from: https://covid19.kemkes.go.id/

6. Dickens BL, Koo JR, Wilder-Smith A, Cook AR. Institutional, not home-based, isolation could contain the COVID-19 outbreak. Lancet [Internet]. 2020;395(10236):1541-2. Available from: $\quad h t t p: / / d x . d o i . o r g / 10.1016 / S 0140-$ 6736(20)31016-3

7. Chen N, Zhou M, Dong X, Qu J, Gong F, Han Y, et al. Epidemiological and clinical characteristics of 99 cases of 2019 novel coronavirus pneumonia in Wuhan, China: a descriptive study. Lancet [Internet]. 2020;395(10223):507-13. Available from: $\quad$ http://dx.doi.org/10.1016/S01406736(20)30211-7

8. Tenda ED, Yulianti $M$, Asaf MM, Yunus RE, Septiyanti W, Wulani V, et al. The importance of chest CT scan in COVID-19. Acta Med Indones. 2020;52(1):68-73.

9. World Health Organization. Regional Office for the Western Pacific. (2020). Algorithm for COVID-19 triage and referral: patient triage and referral for resource-limited settings during community transmission. Manila: WHO Regional Office for the Western Pacific. https://apps.who.int/iris/ handle/10665/331915.

10. Karyono DR, Wicaksana AL. Current prevalence, characteristics, and comorbidities of patients with COVID-19 in Indonesia. J Community Empowerment Health. 2020;3(2):77-84.

11. Zheng Z, Peng F, Xu B, Zhao J, Liu H, Peng J. Risk factors of critical \& mortal COVID-19 cases: a systematic literature review and meta-analysis. J Infect. 2020;81(January).

12. Hu B, Huang S, Yin L. The cytokine storm and COVID-19. J Med Virol [Internet]. 2020;2:0-2. Available from: http://dx.doi.org/10.1002/ jmv. 26232

13. CDC. Symptoms of Coronavirus (COVID-19) [Internet]. Cdc. 2020 [cited 2020 Sep 24]. p. 317142. Available from: https://www.cdc.gov/ coronavirus/2019-ncov/symptoms-testing/ symptoms.html

14. Tobin MJ, Laghi F, Jubran A. Why COVID-19 silent hypoxemia is baffling to physicians. Am J Respir Crit Care Med. 2020;202(3):356-60.

15. Sun X, Wang T, Cai D, Hu Z, Chen J, Liao H, et al. Cytokine storm intervention in the early stages of COVID-19 pneumonia. Cytokine Growth Factor Rev [Internet]. 2020;53(April):38-42. Available from: https://doi.org/10.1016/j. cytogfr.2020.04.002 